\title{
Perturbative reconstruction of a gravitational lens: when mass does not follow light
}

\author{
C. Alard \\ Institut d'Astrophysique de Paris, 98bis boulevard Arago, 75014 Paris, France \\ e-mail: alard@iap.fr \\ Received 7 March 2009 / Accepted 7 July 2009 \\ ABSTRACT

\begin{abstract}
Aims. The structure and potential of a complex gravitational lens is reconstructed using the perturbative method presented in Alard (2007, MNRAS, 382, L58; 2008, MNRAS, 388, 375). This lens is composed of 6 galaxies belonging to a small group. Methods. The lens inversion is reduced to the problem of reconstructing non-degenerate quantities: the 2 fields of the perturbative theory of strong gravitational lenses. Since in the perturbative theory the circular source solution is analytical, the general properties of the perturbative solution can be inferred directly from the data. As a consequence, the reconstruction of the perturbative fields is not affected by degeneracy, and finding the best solution is only a matter of numerical refinement.

Results. The local shape of the potential and density of the lens are inferred from the perturbative solution, revealing the existence of an independent dark component that does not follow light.

Conclusions. The most likely explanation is that the particular shape of the dark halo is due to the merging of cold dark matter halos.
\end{abstract} \\ This is a new result illustrating the structure of dark halos on the scale of galaxies.
}

Key words. gravitational lensing - dark matter

\section{Introduction}

In 1986 Lynds and Petrosian reported the discovery of giant arcs in Abell 370 and 2242-02, with a length of $100 \mathrm{Kpc}$ and luminosities comparable to giant elliptical galaxies, but with bluer colors. Bohdan Paczyński (1987) interpreted these features, he suggested that the arcs may be the result of the clusters acting as gravitational lenses. The Paczyński interpretation was later confirmed by Grossman \& Narayan (1988), and initiated a series of very fruitful developments on gravitational lenses. The lens inversion problem was tackled by Kochanek et al. (1989) using the ring cycle method. Other methods have been developed to solve the lens inversion problem, such as maximum entropy methods by Wallington et al. (1996), methods based on Fourier series expansion (Trotter et al. 2000), LensCLEAN (Wucknitz 2004), semi linear inversion methods (Warren \& Dye 2003), Bayesian methods (Brewer \& Lewis 2006), and potential reconstruction method (Suyu et al. 2009). The lens inversion methods may be classified in 3 types: (i) model dependent reconstructions; (ii) potential reconstruction on a grid; (iii) expansion of the potential in Fourier series. All methods have to consider the degeneracy of modeling, for instance Wayth et al. (2005) found that 6 different models were consistent with the observations for the lens ER 0047-2808. The problem of degeneracy in lens inversion is widespread. The degeneracy issue is related to the fact that the constraints on the potential are local. The value of the potential is constrained only in areas where images of the source are formed. Additional constraints comes from the fact that no images are formed in dark areas (Diego et al. 2005). Since there are no constraints on the potential in other areas, without additional assumptions, the problem is fully degenerate, and an infinite number of models are possible. One possibility is to explore the family of models that is consistent with the observations
(Saha \& Read 2009) and to try to find the common features between the models (non-degenerate quantities). However, in such an approach it is difficult to explore the full range of possible models. Another possibility to reduce the degeneracy is to use a local representation of the potential, like the methods (ii) and (iii). However, the grid methods or Fourier methods require many free parameters, and it is not clear whether the degeneracy problem has been improved. To solve the degeneracy problem it is essential to reduce the number of parameters, and to describe the potential in terms of fundamental, non-degenerate quantities. Since giant arcs resemble a part of a circle, it is natural to consider that arcs corresponds to a small break of the circular symmetry. In such an hypothesis, the fundamental properties of the arcs should be related to the quantities breaking the circular symmetry. These ideas were first explored by Blandford \& Kovner (1988) who demonstrated that for a small impact parameter (with respect to the size of the critical circle) and small deviations from circularity, the equation for caustics depends only on the orthoradial component of the deflection field at the critical circle. Blandford \& Kovner (1988) also presented a geometrical method to reconstruct the images of the lens. The same concepts were investigated later by Alard (2007) who derived an analytical equation to describe the formation of images by the lens. The main advantage of the analytical description is that it directly relates the morphological properties of the arcs to the potential expansion. This relation between the potential and the observational features suggests that the description of the potential is non-degenerate, and also simplifies the lens inversion problem. The reduction of the degeneracy problem in this approach is well illustrated by the fact that an infinite number of models corresponds to a given perturbative representation. Lens inversion in this perturbative framework was already tested in Alard (2008) using numerical simulations. It was found that the 
perturbative approach allows an accurate re-construction of the potential for lenses with substructures. The concepts developed in this approach are very different from some recent perturbative numerical schemes (see for instance Vegetti \& Koopmans 2009). In this type of work there is no attempt at reformulating the lens-inversion problem in terms of fundamental quantities; the main goal is to derive an efficient numerical scheme to solve the ordinary lens equation. Peirani et al. (2008) showed that the perturbative approximation is accurate for realistic lens models. The numerical accuracy is of about $1 \%$ in units of the critical radius. The lens model of Peirani et al. (2008) were extracted from cold dark matter simulations (Horizon project), and some of them have a lot of structure (merging). This paper is organized as follows: the first part will present the perturbative method, and its basic properties. In an effort to make this work self-contained, a synthesis of the other useful results on the pertubative method is presented in the appendices. This section also has the advantage of presenting all the equations essential to lens inversion. The second part of the paper will present the application of the perturbative method to the inversion of an interesting case of gravitational lens. This paper also will be an occasion to extend the technique of perturbative inversion and to relate the perturbative fields to the geometry of the lens.

\section{The perturbative approach in gravitational lensing}

In the perturbative approach (Blandford \& Kovner 1988; Alard 2007), gravitational arcs are interpreted as a perturbation of the perfect ring situation. A perfect ring is formed when a point source is situated at the center of a circularly symmetric lens. In the perfect ring situation, the central point has an infinite number of images located on the critical circle. There are 2 different kinds of perturbations that breaks the symmetry of the system: the non-circularity of the lens, and the source impact parameter. When such non-circular perturbations are introduced, the symmetry of the system is lost and the ring is replaced with a series of individual images. However, as illustrated in Alard (2007) whatever the angular position $\theta$ of these perturbed images, there is always an unperturbed image of the central point on the critical circle at the same angular position. As a consequence there is only a radial displacement $\mathrm{d} r$ between the perturbed and un-perturbed images. The un-perturbed situation is represented by a circular lens with potential $\phi_{0}(r)$, and a point source with null impact parameter. In the perturbed situation, the source has an impact parameter $\boldsymbol{r}_{\mathrm{S}}$, and the lens is perturbed by the non-circular potential $\psi(r, \theta)$. Both perturbations $\boldsymbol{r}_{\mathrm{S}}$ and $\psi$ are assumed to be of the same order $\epsilon$ :

$$
\left\{\begin{array}{l}
r_{\mathrm{S}}=\epsilon \boldsymbol{r}_{\mathrm{S}} \\
\phi=\phi_{0}+\epsilon \psi
\end{array}\right.
$$

Note that for convenience the unit of the coordinate is the critical radius. Thus by definition in this coordinate system the critical radius is situated at $r=1$. As a consequence, in the continuation of this work all distances and their associated quantities (errors,..) will be expressed in units of the critical radius. In response to this perturbation, the radial position of the image will be shifted by an amount $\mathrm{d} r$ with respect to the unperturbed point on the critical circle. The new radial position of the image is:

$r=1+\epsilon \mathrm{d} r$.
The response $\mathrm{d} r$ to the perturbation can be evaluated by solving the lens equation in the perturbative regime. The lens equation reads:

$\boldsymbol{r}_{\mathrm{s}}=\left(r-\frac{\partial \phi}{\partial r}\right) \boldsymbol{u}_{r}-\left(\frac{1}{r} \frac{\partial \phi}{\partial \theta}\right) \boldsymbol{u}_{\theta}$

The last step to solve the lens equation is to expand the potential is series of $\epsilon$ :

$\phi=\phi_{0}+\epsilon \psi=\sum_{n=0}^{\infty}\left[C_{n}+\epsilon f_{n}(\theta)\right](r-1)^{n}$

with:

$\left\{\begin{array}{l}C_{n}=\frac{1}{n !}\left[\frac{\mathrm{d}^{n} \phi_{0}}{\mathrm{~d} r^{n}}\right]_{(r=1)} \\ f_{n}(\theta)=\frac{1}{n !}\left[\frac{\partial^{n} \psi}{\partial r^{n}}\right]_{(r=1)} .\end{array}\right.$

By inserting Eqs. (1), (2), and (4) in the lens equation (Eq. (3)), it is straightforward to obtain an equation relating the response $\mathrm{d} r$ to the perturbation $r_{\mathrm{S}}$ and $\psi$ :

$\boldsymbol{r}_{\mathrm{s}}=\left(\kappa_{2} \mathrm{~d} r-f_{1}\right) \boldsymbol{u}_{r}-\frac{\mathrm{d} f_{0}}{\mathrm{~d} \theta} \boldsymbol{u}_{\theta}$

and $\kappa_{2}=1-\left[\frac{\mathrm{d}^{2} \phi_{0}}{\mathrm{~d} r^{2}}\right]_{(r=1)}$.

This equation corresponds to Eq. (8) in Alard (2007).

Considering that the source has a mean impact parameter $\boldsymbol{r}_{0}$, the position in the source plane may be re-written: $\boldsymbol{r}_{\mathrm{S}}=\tilde{r_{\mathrm{S}}}+r_{0}$. Assuming Cartesian coordinates $\left(x_{0}, y_{0}\right)$ for the vector $\boldsymbol{r}_{0}$, Eq. (6) reads:

$\tilde{\boldsymbol{r}}_{\mathrm{s}}=\left(\kappa_{2} \mathrm{~d} r-\tilde{f}_{1}\right) \boldsymbol{u}_{r}-\frac{\mathrm{d} \tilde{f}_{0}}{\mathrm{~d} \theta} \boldsymbol{u}_{\theta}$

For a circular source with radius $R_{0}$, the perturbative response $\mathrm{d} r$ takes the simple following form (Alard 2007, Eq. (12)):

$\mathrm{d} r=\frac{1}{\kappa_{2}}\left[\tilde{f}_{1} \pm \sqrt{R_{0}^{2}-\left(\frac{\mathrm{d} \tilde{f}_{0}}{\mathrm{~d} \theta}\right)^{2}}\right]$.

Equation (6) depends on $\kappa_{2}$. However this variable can be eliminated from Eq. (6) by re-normalizing the fields: $f_{n}=\frac{f_{n}}{\kappa_{2}}$, and the source plane coordinates, $\boldsymbol{r}_{\mathrm{s}}=\frac{\boldsymbol{r}_{\mathrm{s}}}{\kappa_{2}}$ (mass sheet degeneracy). These re-normalized variables will be adopted in the rest of this work. The re-normalization is equivalent to solving Eqs. (6) and $(8)$ for $\kappa_{2}=1$. The variable $\kappa_{2}$ will re-appear when the renormalized quantities are converted to the original quantities.

\section{Pre-processing of the HST data}

The gravitational lens SL2SJ02140-0532 (Cabanac et al. 2007, SL2S public domain) was observed by HST in 3 spectral domains, F475W, F606W, F814W, with an exposure time of $400 \mathrm{~s}$. The main difficulty in the pre-processing of the images is to remove the large number of cosmic present in the images. Cosmic are identified using a wavelet approach based on an estimation of the local scale in the image. Once a cosmic has been identified at a given position, the local area around this position is investigated to remove any other pixels connected to the cosmic. This procedure is based on routines from the ISIS package (Alard 2000). Note that since the number of cosmic is large in the images, the local density of cosmic sometime become too large in 


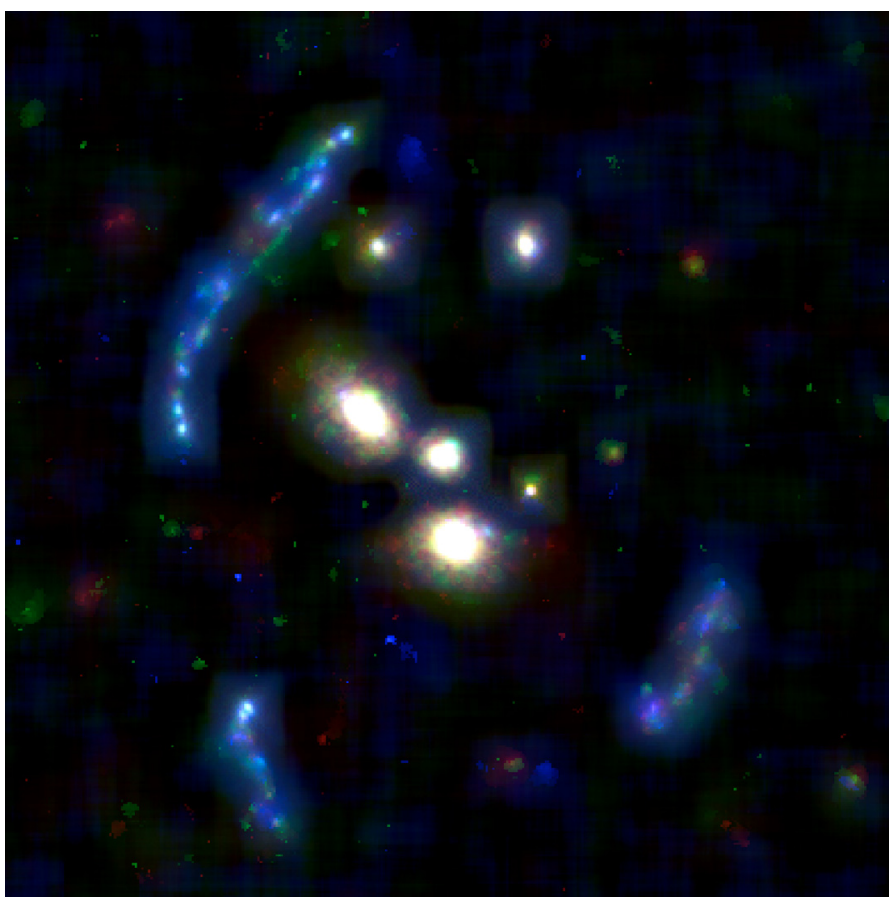

Fig. 1. Color image for SL2SJ02140-0532 reconstructed from 3 noise filtered HST images in 3 bands.

some areas. When too many nearby cosmic are detected within a small area, the area is flagged, and will be considered cautiously, or even rejected. Once the 3 images are cosmic cleaned, a re-centering to a common grid is performed using the position of the bright objects. The last step in the processing of the images is to remove the background. There is some diffuse light coming from the galaxy halos at the level of the arcs, and the proper removal of this diffuse light is especially important. The diffuse background is removed in several steps. The first step is to build a general background model. To build this model, the image is divided in square areas of 32 pixel, the local background is estimated within each area using a $\kappa \sigma$ cleaning method. The background value in each local area is then interpolated to compute a general background value at each position in the image. Once this background model has been subtracted from the image, the residuals around the arcs are investigated in order to control any systematic errors. A tiny systematic background residual is detected; this residual depends on the distance to the 3 main galaxies center of gravity. This background residual is related to an imperfect subtraction of a diffuse halo centered around the 3 main galaxies. An elliptical component centered at the center of gravity of the 3 galaxies is fitted to this residual. The best elliptical parameters are estimated by minimizing the deviations to elliptical symmetry. The mean radial profile of the residual is estimated, and finally the relevant elliptical component is subtracted. Note that this last background correction is small, it is only a few percent of the initial background subtraction. Thus, the 3 cosmic cleaned, re-centered and background subtracted images are stacked, to produce the final reference image of the arc system. A color illustration of the arc system is provided in Fig. 1.

\section{Lens inversion}

This work presents the first successful model of the images formed by the lens SL2SJ02140-0532. This system was investigated by Limousin et al. (2009), who tried to fit this system using an elliptical lens model. They found that it was not possible to reproduce this system using such simple elliptical models. Limousin et al. (2009) were also confused by the color of the last image (right side in Fig. 1) which is slightly different to the color of the other images. They concluded that the identification of the images formed by this lens was uncertain, and as a consequence renounced modelling of this system. We will see in the continuation of this work that this lens requires models much more complex than elliptical, and that such models can be built naturally using the perturbative method presented in this paper. The development of this perturbative model also will naturally solve the problem of the different color of the last image. We will see that the first part of this image has the same color as the other images, while the remaining part of the image has a different color. This is a direct prediction of the perturbative model, which offers an elegant solution to the problems encountered by Limousin et al. (2009). We now turn to the analysis of this lens using the perturbative method. The lens SL2SJ021408-053532 forms a system of 4 images of the source. The first 2 images are situated inside the large arc in the upper left (they appear as 2 identical mirror images). The 2 other image, are at the bottom and the right side of the frame (see Fig. 1). In the perturbative approach the problem of reconstructing this lens is equivalent to the reconstruction of the perturbative fields, and the next sections will concentrate on this issue. Obviously, the re-construction problem is reduced to the reconstruction of the first order perturbative field only if the first order perturbative theory applies. The requirement for this approximation to work is that the gradient of the potential can be linearized in the vicinity of the critical circle (Alard 2007). For single dark matter halos numerical experiments shows that this local constraint on the gradient is usually met with good accuracy (Alard 2007; Peirani et al. 2008). It is only in the case where a local minima of the potential is present close to the critical circle that this approximation may not work. But such situations are very rare because they require that for instance a sub-structure falls very close to the critical line. However Alard (2008) showed that the general treatment of substructure in this approximation is correct even when the substructure is quite close to the critical circle (up to one tenth of the critical circle). Additionally, when an unlikely situation happens, such as a substructure lying on the critical line, the consequences are easily observable: a few additional images are formed near the perturbator. Thus such situation is easy to recognize, and is not apparent in the present data. In any case the best test of the first order approximation is to perform a successful reconstruction of the data, which means that in practise second order terms can be neglected. This will be the approach favored in this paper. Before the field re-construction problem can be tackled, a necessary step is to evaluate the size of the critical circle, which will become by convention the coordinate system unit.

\subsection{Estimation of the critical circle}

The critical circle is estimated by fitting a circle to the mean position of the images. The center and radius of the circle are adjusted. The non-linear adjustment procedure starts from a circle centered on the small galaxy at the center of the image. The initial estimate for the circle radius is the mean distance of the images to the center. A non linear optimization shows that the best center is close to the initial guess and that the optimal radius is close to 152 pixels; with a pixel size of $0.049^{\prime \prime}$ the critical circle radius $R_{\mathrm{C}}$ is $7.44^{\prime \prime}$. This value should be compared to the radius of 7.31" found by Limousin et al. for the same lens. Note 
that the final result will not depend upon the particular choice of a given critical circle. Taking another circle close to this one would change a little the estimation of the perturbative fields, but the total background plus perturbation would remain the same.

\subsection{General properties of the solution}

The general properties of the solution can be derived from the properties of the circular source solution. To estimate the circular source solution, the outer contour of the source will be approximated with a circle. This approximation of the source may not be very accurate; the typical error on the field estimation will be of the order of the source outer contour deviations from circularity. However, this approximation is sufficient to obtain a first estimate of the solution and to derive its general properties. For circular sources the perturbative fields are directly related to the data (see Appendices A, and Eq. (8)). This direct relation between the fields and the data is possible only in areas of the lens plane where images of the source are present. In dark areas, the field will have to be reconstructed by interpolating the values in bright areas. The field $\tilde{f}_{1}$ will be approximated with the mean radial position of the images (with respect to the critical circle). The reconstruction of $\tilde{f}_{1}$ is simple and not ambiguous, but the reconstruction of $\frac{\mathrm{d} \tilde{f}_{0}}{\mathrm{~d} \theta}$ requires more work. On the other hand $\frac{\mathrm{d} \tilde{f}_{0}}{\mathrm{~d} \theta}$ is better constrained since no images should be formed in dark areas $\left(\left|\frac{\mathrm{d} \tilde{f}_{0}}{\mathrm{~d} \theta}\right|>R_{0}\right)$. Furthermore, the field $\frac{\mathrm{d} \tilde{f}_{0}}{\mathrm{~d} \theta}$ is of particular interest since it is directly related to the potential iso-contour (Eq. (C.1)). The derivation of the general properties of $\frac{d \tilde{f}_{0}}{\mathrm{~d} \theta}$ require some specific guess of the local behavior of this field in the region of image formation. Images form in minima of $\left|\frac{d \tilde{f}_{0}}{\mathrm{~d} \theta}\right|$ and the example presented in Appendix A indicates that the behavior of $\frac{\mathrm{d} \tilde{\tilde{f}_{0}}}{\mathrm{~d} \theta}$ near the minima is of 2 types. For small images $\frac{d \tilde{f}_{0}}{d \theta}$ has a linear behavior, while for larger images (caustics) the field behaves like a higher order polynomial. In the case of this particular lens system, we have 4 images of comparable size. The typical image size is quite small and it is reasonable to assume that the field behavior in the image is linear. Of course other models could be investigated, with for instance order 2 behavior of the field, but obviously these models are much more complex and would require higher order Fourier expansions (see Fig. 2). Additionally, adopting a higher order description (lower curve in Fig. 2) may introduce false features like local peaks. The locally linear model (upper curve in Fig. 2) is the simplest description consistent with the general properties of $\frac{\mathrm{d} \tilde{f_{0}}}{\mathrm{~d} \theta}$; it has the smallest possible number of minima and maxima. Thus adopting the locally linear model guarantees that no un-necessary features are introduced in the model. On the other hand, in reality this description may be too simple. The validity of this model can only be tested by a direct application to the data, and an evaluation of the quality of the fit. A higher order description should be adopted only if the locally linear model does not fit the data properly. As a consequence, the locally linear model will be adopted in the continuation of this work. Once the general shape of the solution is known, everything is now just a matter of improving the numerical accuracy. The process of building the solution will proceed in several steps. First the local solution will be refined numerically, then some interpolation of the solution in dark areas will be computed. A Fourier expansion will be fitted to this piecewise polynomial solution. And finally the adjustment of the Fourier expansion to the data will be optimized using a non linear minimization procedure. Note that since only the square of $\frac{\mathrm{d} \tilde{f}_{0}}{\mathrm{~d} \theta}$ enters

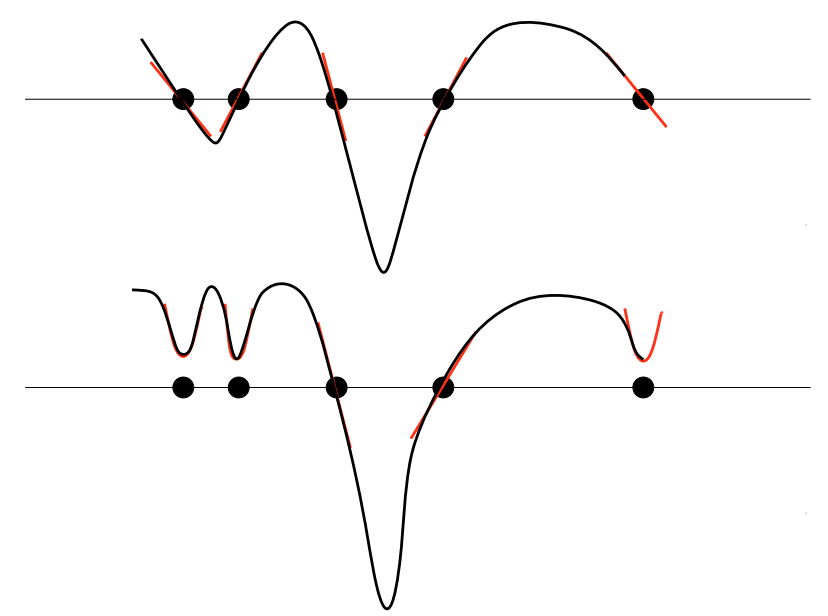

Fig. 2. Two example of possible shapes for the field $\frac{\mathrm{d} \tilde{f}_{0}}{\mathrm{~d} \theta}$. The black dots represents the mean image position. The red curve represents the local behavior of the field near the image, while the black curve is a smooth extrapolation. The upper curve present a solution where the field has local linear behavior near the images. In the lower curve a higher order behavior is presented. In dark areas the field must be larger that the source radius to avoid forming images (see Eq. (8)). Note also that the field $\frac{\mathrm{d} \tilde{f}_{0}}{\mathrm{~d} \theta}$ must also have zero sum.

Eq. (8), the sign of $\frac{\mathrm{d} \tilde{\tilde{f}_{0}}}{\mathrm{~d} \theta}$ is not known, as a consequence two solutions with different sign will have to be explored. Hopefully, when the source is not circular this degeneracy of the sign can be broken.

\subsection{Refinement of the local solution}

The study of the general properties of $\frac{\mathrm{d} \tilde{f}_{0}}{\mathrm{~d} \theta}$ indicates that the simplest model of this field is locally linear in the neighborhood of the images. This local linear model will be refined in this section. The same local linear approximation will be used for the field $\tilde{f}_{1}$. As illustrated in the former section, the estimation will start from the circular source guess. For a circular source and a linear field model, the field $\frac{\mathrm{d} \tilde{f}_{0}}{\mathrm{~d} \theta}$ is zero at the center of the image. This property will be assumed in the refinement of the local solution. The slope of the linear model for $\frac{d \tilde{f}_{0}}{\mathrm{~d} \theta}$ is estimated by solving Eq. (8). Note that solving Eq. (8) requires an estimation of the source radius $R_{0}$. Since the image maximum half radial width is $R_{0}$, an estimation of $R_{0}$ can be obtained by averaging the maximum radial width of the 4 images. By adopting this linear local model of $\frac{\mathrm{d} \tilde{f}_{0}}{\mathrm{~d} \theta}$, taking the mean radial position as an estimation of $\tilde{f}_{1}$ linear parameters, and using the perturbative lens equation (Eq. (6)), it is possible to fold the 4 images to the source plane. Once folded to the source plane, the 4 images must be similar and their comparison is a direct test of the quality of the lens re-construction. With the initial guess for the field a, correlation between the 4 folded images is effectively observed: the mean cross-correlation between the images is 0.6. This cross correlation can be improved quite significantly by non-linear optimization. The cross-correlation between images in the source plane is maximized using the Nelder \& Mead (1965) simplex method. The parameter space of the maximization is the 4 local slopes of the $\frac{\mathrm{d} \tilde{f}_{0}}{\mathrm{~d} \theta}$ field, and also the 4 local slopes of the field $\tilde{f}_{1}$. The simplex maximization brings the mean cross-correlation between images to 0.8 which is a good indication that the linear approximation is already a good model. This maximization 


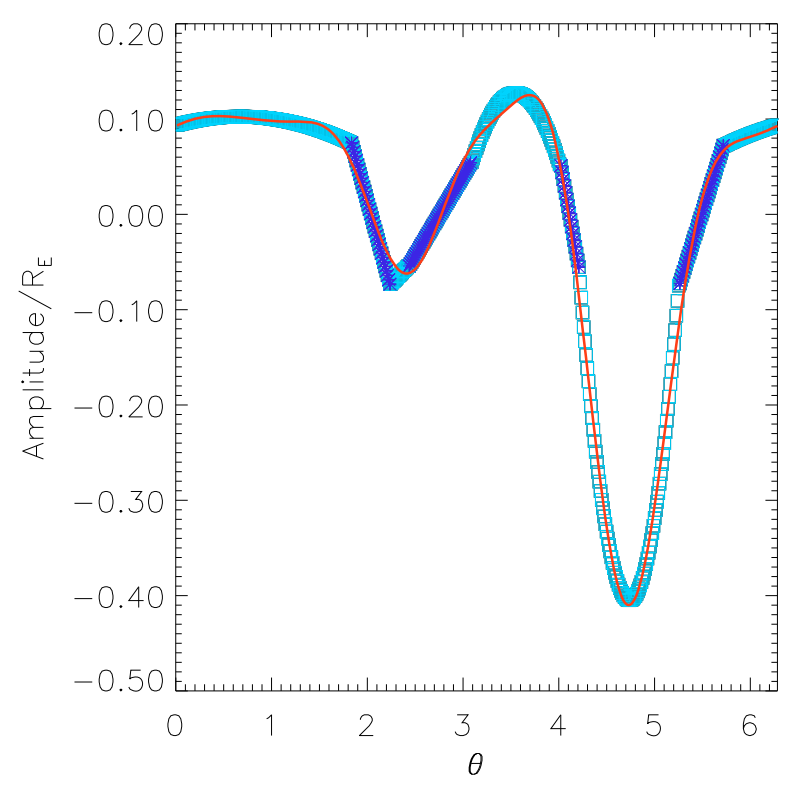

Fig. 3. An estimate of the $\frac{\tilde{\mathrm{d}} f_{0}}{\mathrm{~d} \theta}$ field. The blue line segments represent the local linear guess at the image location, while the light blue curves are the second order interpolations between images. The red line is an adjustment of a 6th order Fourier series to the blue curve.

is also the occasion to build a first realistic model of the source, and to go beyond the circular source approximation. The result for the field $\frac{\mathrm{d} \tilde{f}_{0}}{\mathrm{~d} \theta}$ is presented in Fig. (3). The local linear approximation is the dark blue line segments in Fig. (3).

\subsection{Interpolating the local linear solution in dark areas}

Since there are no constraints on the field $\tilde{f}_{1}$ in dark areas, the fit of this field in the full angular range is straightforward. Due to the constraint that no images are formed in dark areas, the situation is more complicated for the field $\frac{\mathrm{d} \tilde{f}_{0}}{\mathrm{~d} \theta}$. The local linear solution obtained in the former section needs to to be completed with second order polynomials to fill the gaps between the images. The condition that no images are formed in dark areas (Diego et al. 2005) is equivalent to the condition that the functional $\left|\frac{\mathrm{d} \tilde{f}_{0}}{\mathrm{~d} \theta}\right|$ is larger in dark areas than in areas of image formation. Another constraint is that the sum of the field $\frac{\mathrm{d} \tilde{f_{0}}}{\mathrm{~d} \theta}$ must be zero. And finally the field must be continuous and as smooth as possible. The first constraint is a constraint on the sign of the curvature of the local polynomial, or equivalently on the sign of the second order coefficient of the polynomial. The second constraint reduces the number of unknowns in the polynomial coefficients. And the last constraint settles the value of the remaining coefficients. Note that the constraints on zero and first order polynomial coefficients can be solved using linear methods, which reduce the number of unknown and leaves us with variables depending only on the second order coefficients. The remaining equations depending on the second order coefficients have to be solved with a constraint on the sign of the coefficients. A simple grid method was adopted to solve the relevant equations. The final solution is presented in Fig. 3 (light blue curve).

\subsection{Final fitting of the fields}

The final fitting of the fields will be conducted by reconstructing the images of the source and comparing these images with the HST data by chi-square estimation. This procedure will take into account the convolution of the images with the PSF, which was ignored in the former reconstructions. The HST PSF is estimated using the Tiny Tim software (Krist 1995). Note that the chi-square value between model and data can be interpreted as a comparison between the images. In the model, all images come from the same source, thus the chi-square directly measures the image similarity in the lens plane. In practice, the fitting procedure will work in the following way: start from a numerical guess of the field, reconstruct the source, then reconstruct the images of the source, and finally estimate the chi-square with respect to the HST data. An essential component in this procedure is the source reconstruction method, which is illustrated in the next section.

\subsubsection{Source reconstruction}

At this point the simplest method to estimate the source would be to use the Warren \& Dye (2003) method. Unfortunately the source is very complex, and this method would require using too many basis functions, which in practice is un-tractable. The following method has been preferred: images are co-added in the source plane using an algorithm that preserves the geometrical properties of the images elements. The basic idea of this geometrical method is that pixels in the lens plane are divided in 2 triangles. These triangles are transported to the source plane and are assumed to remain triangles. The triangles in the source plane are projected on a grid and the flux is co-added. The optimal size of the grid is estimated by numerical experiments. The noise level in the source image is estimated by looking at a specific area, and only the significant pixels in the source grid are kept. The images of the source in the lens plane are computed using the same geometrical method, but this time in the opposite direction. The final step is to compare the images of the source with the data. The drawback of this method is that the pixels are affected by the convolution with the PSF, and thus the source reconstruction is not perfect. However, it is easy to correct this effect by using the following procedure: first ignore the effect of the PSF on source reconstruction, find a solution, and then correct the effect using a deconvolution procedure very similar to the Van-Cittert method (Van Cittert 1931). This deconvolution of the source starts from the solution obtained ignoring the PSF convolution. The initial guess is convolved with the PSF and a difference image is formed by subtracting the data. This difference image is taken as a new input to the source reconstruction procedure in order to produce a first correction of the initial guess of the source. This procedure is further iterated until only noise is present in the difference image. In practice this method requires to control the noise in the difference image. In this work a 3- $\sigma$ level was adopted; tests were performed with other thresholds, 2 and $4 \sigma$, with no significant change in the results. The lower $\sigma$ cuts require more iterations and provide somewhat more noisy source re-construction. However, since the areas where effective corrections are needed are quite small (these areas correspond to the bright sharp features in the images), the source reconstruction is not affected much. It is also important to note that these PSF corrections have very little effect on the re-construction of the perturbative fields. By comparing the fit obtained by ignoring the PSF and correcting for the PSF, it was found that the difference is only a small fraction of the noise in the field re-construction. Thus for this particular lens this procedure of PSF de-convolution is important only for the re-construction of the source. This is due to the fact that for this particular lens the thickness of the arc system is quite large with 
respect to the PSF size, and as a consequence the PSF convolution has only a limited effect.

\subsubsection{Fitting of a Fourier model}

The practical implementation of the fitting procedure presented above now will be described. The first step is to choose a starting estimate. It could be the piecewise polynomial model presented in Sect. 4.6, but since the Fourier expansion of the fields is directly related to the multipole expansion of the potential (see Eqs. (B.1) (B.2) and (B.3)), it is more convenient to use Fourier series. The initial guess is obtained by fitting a 6th order Fourier series to the piecewise model of $\frac{\mathrm{d} \tilde{f}_{0}}{\mathrm{~d} \theta}$, and for $\tilde{f}_{1}$ by fitting a Fourier series to the mean radial position of the images. Starting from this initial guess, the source and image reconstruction procedures are iterated using the simplex method, in order to minimize the chi-square between the model and data. This procedure converges to the minimum of the chi-square in the parameter space. This minimum corresponds to the optimal Fourier coefficients. Note that as pointed out in Sect. 4.2, the sign of the initial guess is unknown and 2 solutions with different signs have been explored. Fortunately in this case the source is not circular and the 2 solutions have different chi-square, which solves the problem of the degeneracy of the solution sign. The final solution, the source reconstruction and the image reconstruction are presented in Fig. 5, and the field solution is presented in Fig. 4. The fact that the source is complex and has a number of small features (see Fig. 7) can be used to estimate the accuracy of the modeling. There are 3 bright features in the source that are visible in all images (red, yellow, blue in Figs. 6 and 7). The position of these features in the HST data and model has been compared for each image. To obtain the best possible accuracy in the comparison of the positions, the following procedure was used: a small image of each feature is extracted in the model frame, and this small image is cross-correlated with the HST data at various positions around the feature. The cross-correlation map obtained using this procedure is fitted with a polynomial to locate the map maxima, to derive a direct estimate of the shift between the features. The residuals are somewhat smaller for the first 2 images. In particular a small discrepancy is visible in the bright part of the third image (lower left image); the distance between the two images of the bright features in the source are closer in the model. The discrepancy is of about 2.4 pixels, which is about $1.6 \%$ of the critical radius. On average the mismatch between model and observations is $0.9 \%$ of $R_{\mathrm{C}}$. This estimation is very close to the results obtained by Peirani et al. (2008) who found that for realistic lens models the mean errors on the reconstruction of images using the perturbative approximation was about $1 \%$ of $R_{\mathrm{C}}$. Note that two other features are visible in the source (see Fig. 7); due to variable levels of amplification, noise, and blurring by other nearby features, the images of these features are not always clearly visible in the HST data. However, when they are visible in the images, their position and the correspondence between model and data are indicated in Fig. 6. Note that the caustic system associated with the solution is complex and includes a number of sharp features (see Fig. 9). The complexity of this caustic system is not easily visible in the images; this is due to the fact that the source is large with respect to the small features in the caustic system.

\subsection{Noise}

The model and the data are compared in a small region around the arcs with total number of pixels $N$. The Poisson weighted

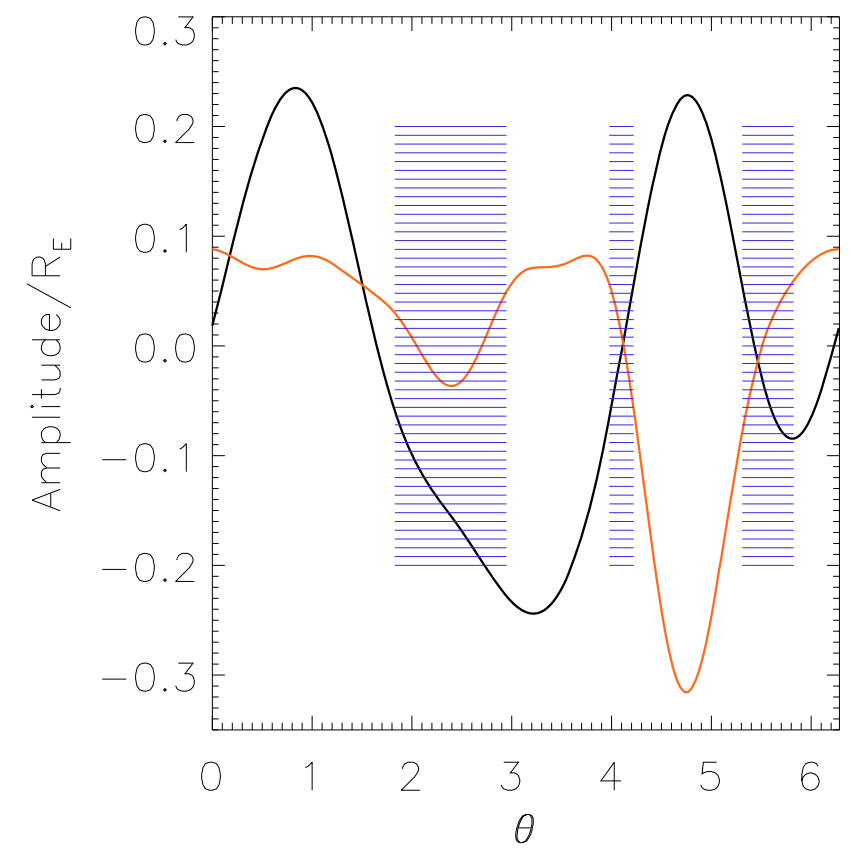

Fig. 4. Best solution for the fields: red curve, $\frac{\tilde{\mathrm{d}} f_{0}}{\mathrm{~d} \theta}$, and black curve $\tilde{\widetilde{f}}_{1}$. The blue dashes indicates areas where images are present.

difference $R_{i}$ between the model at pixel $i, M_{i}$ and the HST data $D_{i}$ is very close to a Gaussian (see Fig. 8). Considering that the model has $N_{P}$ parameters, 25 parameters of the Fourier expansion of the fields, and the approximately 450 pixels of the source, the chi-square is, $\chi_{2}$ /d.o.f. $=\frac{1}{N-N_{\mathrm{P}}} \sum_{i} R_{i}^{2} \simeq 1.17$. Changing a little the size of the area, either reducing it by moving closer to the center of the arcs, or enlarging the area does not significantly change the chi-square value.

\subsubsection{Errors on the Fourier coefficients due to noise in the data}

The errors on the estimation of the Fourier coefficients are evaluated by local linearization methods near the minima (see, for instance Press et al. 2007). The intensity $M_{i}$ is a function of the $N_{\mathrm{F}}$ Fourier parameters $p_{n}, M_{i}=M_{i}\left(p_{n}\right)$. Assuming that $p_{n}$ is very close to the solution and adding a small shift to the parameters $\mathrm{d} p_{n}$, the intensity variation can be linearized.

$\mathrm{d} M_{i}=\sum_{n} \frac{\partial M_{i}}{\partial p_{n}} \mathrm{~d} p_{n}$

The parameters $\mathrm{d} p_{n}$ can be estimated by a linear least-square fit from Eq. (9), and thus the relevant errors are also estimated by taking the diagonal elements of $A=B^{-1}$, the normal leastsquare matrix invert $B, B_{n m}=\sum_{i, j} \frac{1}{\sigma_{i} \sigma_{j}}\left[\frac{\partial M_{i}}{\partial p_{n}}\right]\left[\frac{\partial M_{j}}{\partial p_{m}}\right]$. This error estimation indicates that the errors on the Fourier coefficients due to the Poisson noise are $\simeq 0.5 \times 10^{-3}$.

\subsubsection{Errors on the reconstruction of the perturbative fields}

In the former section it was shown that the accuracy in the reconstruction of the images was close to $0.9 \%$ of $R_{\mathrm{C}}$. This error is directly related to the errors on the reconstruction of the perturbative fields. To estimate the amplitude of the errors on the fields it is particularly useful to consider circular sources. 

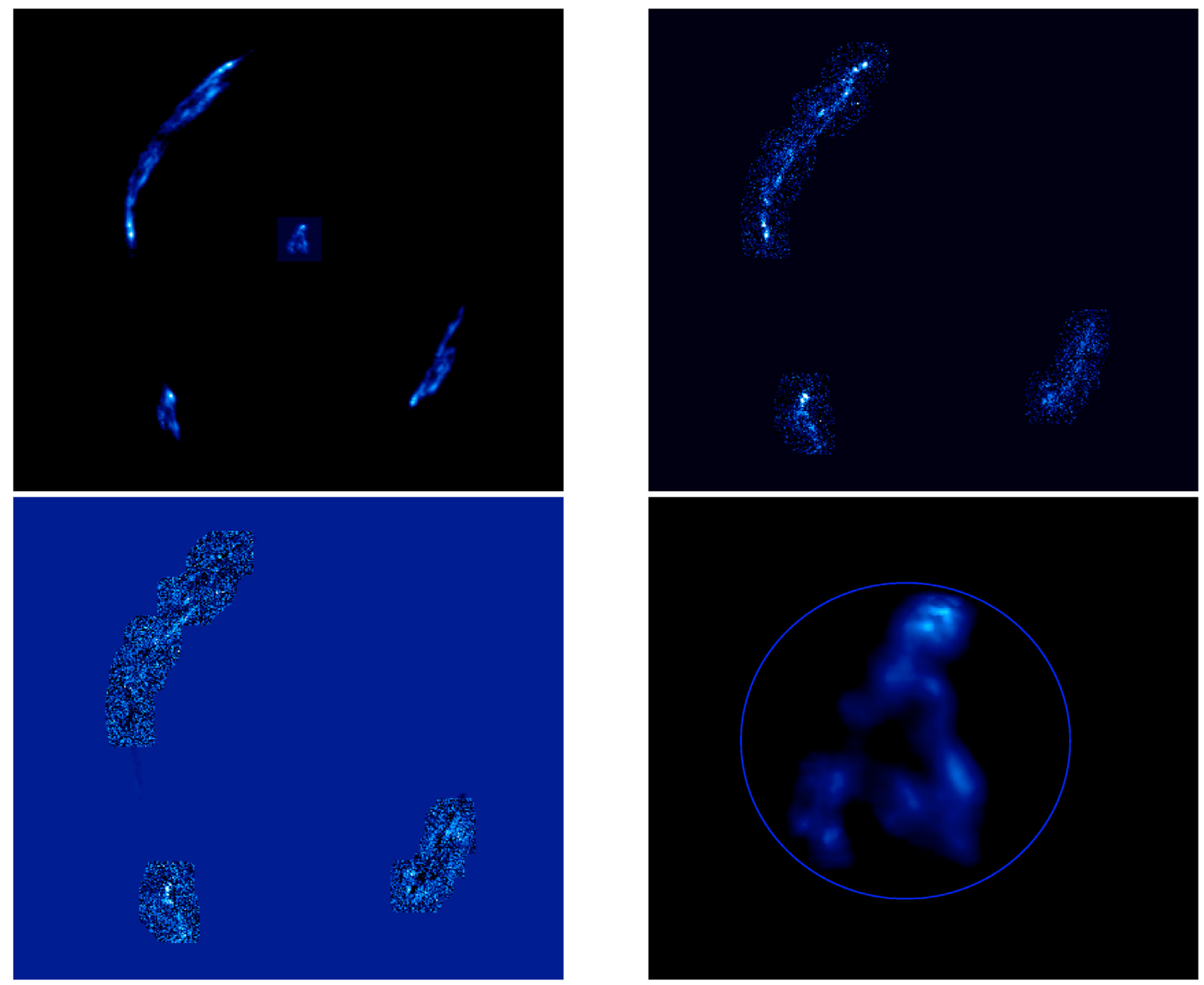

Fig. 5. Results from image re-construction: HST data (upper right), model (upper left), data and model subtraction (lower left), source (lowerright). Note the reproduction of the source details in the images of the model. An image of the source has been super-imposed on the model (upper left), to illustrate the scaling.

For a circular source there is a direct relation between the errors on the fields and the errors on image reconstruction. Eq. (8) shows that for circular sources, $\tilde{f}_{1}$ is the mean radial position of the image. As a consequence, the errors on $\tilde{f}_{1}$ are of the same order as the errors on the image position, which were estimated to be of the order of a percent. The field $\frac{\mathrm{d} \tilde{f}_{0}}{\mathrm{~d} \theta}$ is related by Eq. (8) to the radial width $W$ of the image:

$W^{2}=R_{0}^{2}-\left(\frac{\mathrm{d} \tilde{f}_{0}}{\mathrm{~d} \theta}\right)^{2}$.

As a consequence, the error on $W$ and the error on $\frac{\mathrm{d} \tilde{f}_{0}}{\mathrm{~d} \theta}$ are related:

$\delta W^{2}=\delta\left(\frac{\mathrm{d} \tilde{f_{0}}}{\mathrm{~d} \theta}\right)^{2}$.

Assuming the statistics of the variable $W$ and $\frac{\mathrm{d} \tilde{f}_{0}}{\mathrm{~d} \theta}$ are the same and differ only by a scale factor, the above equation shows that this scale factor must be unity. As a consequence, the errors on $\frac{\mathrm{d} f_{0}}{\mathrm{~d}}$ are of the same order as the errors on the image radial width $W$, which are similar to the errors on image reconstruction: $\simeq 1 \%$. The corresponding errors on the Fourier coefficients of the field expansion of order $m$ can be estimated by analyzing the variance of the expansion:

$F_{i}=\sum_{j=1}^{m} a_{j} \cos (j \theta)+b_{j} \sin (j \theta)$.

Note that here $F_{0}=\frac{\mathrm{d} \tilde{f}_{0}}{\mathrm{~d} \theta}$ and $F_{1}=\tilde{f}_{1}$. Taking the square of $F_{i}$ and averaging over $\theta$

$\frac{1}{2 \pi} \int F_{i}^{2} \mathrm{~d} \theta=\sum_{j=1}^{m} \frac{a_{j}^{2}}{2}+\frac{b_{j}^{2}}{2}$.

Provided it is assumed that the variance of $\left\langle a_{j}^{2}\right\rangle=\left\langle b_{j}^{2}\right\rangle=\sigma^{2}$, the variance of $F_{i}$ reads

$\left\langle F_{i}^{2}\right\rangle=m \sigma^{2} \simeq 0.01^{2}$.

Note that if the Fourier expansion has no constant term, the mean value of $F_{i}$ is zero $\left(\int F_{i} \mathrm{~d} \theta=0\right)$. In practice there is an additional constant term for the field $f_{1}$, however considering that the number of Fourier parameters is quite large (12 parameters), the variance due to this additional parameter will be neglected. As a consequence for both fields the errors on the Fourier coefficients is $\sigma \simeq 0.004$. This error is about 10 times larger than the 

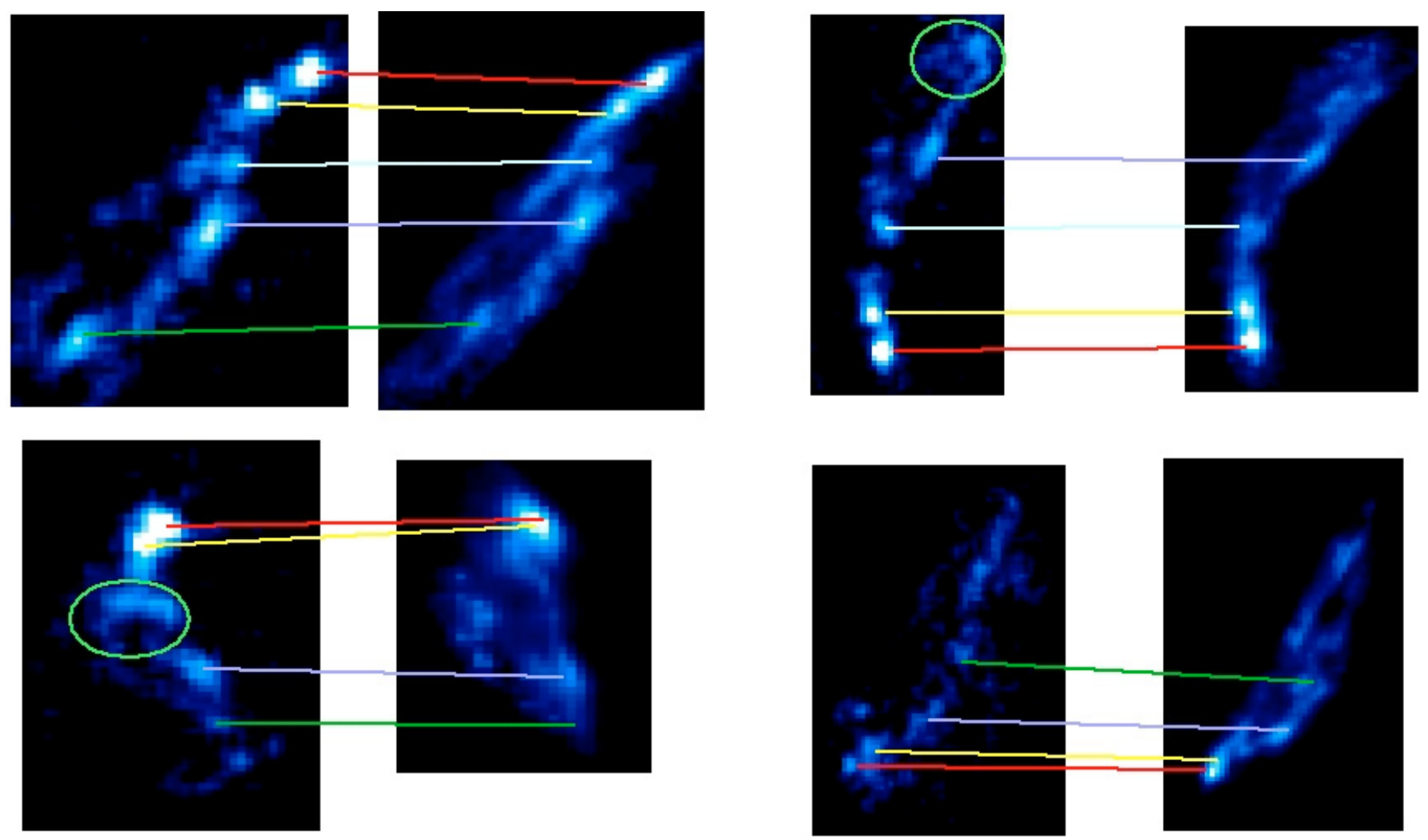

Fig. 6. Correspondence between the features in the model (right side) and the HST data (left side). The color code of the relevant source feature is presented in Fig. 7. The green ellipses indicate areas where cosmic cleaning was not satisfactory. There are a very large number of cosmic in the images, and sometimes several of them falls nearly in the same area, making cosmic cleaning very difficult.

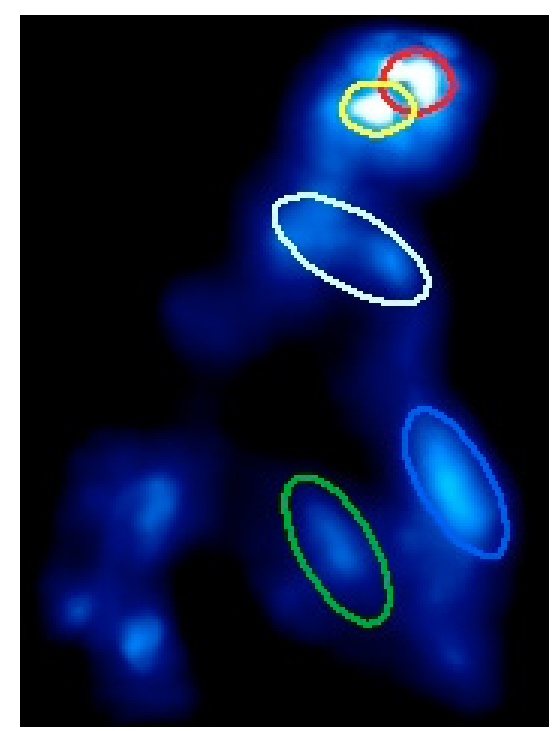

Fig. 7. The source features visible in the images (see Fig. 7). The 3 brightest features are outlined by red, yellow, and blue ellipses. These features are used to estimate the accuracy of the reconstruction. The part of the source at the lower left of the image is effectively visible only in one of the images (see Sect. 5 for more explanations).

error due to the Poissonian noise in the image, which is clearly negligible. As a consequence, in the continuation of this work, only the noise due to the perturbative approximation will be considered.

\subsubsection{Effects of the errors on the potential and density estimation}

Some interesting properties of the lens are directly related to the perturbative fields as illustrated in Eqs. (C.1) and (D.2). The potential iso-contour depends on $f_{0}$, and it will be assumed that the variance of $f_{0}$ is identical to the variance of $\tilde{f}_{0}$. The variance on $\tilde{f}_{0}$ can be estimated using the method presented in Sect. 4.6.2. The expansion for $\tilde{f}_{0}$ read:

$\frac{\mathrm{d} \tilde{f}_{0}}{\mathrm{~d} \theta}=\sum_{j=1}^{m} a_{j} \cos (j \theta)+b_{j} \sin (j \theta)$.

Thus

$\tilde{f_{0}}=\sum_{j=1}^{m} \frac{a_{j}}{j} \cos (j \theta)+\frac{b_{j}}{j} \sin (j \theta)$.

Using Eq. (10) the associated variance of $\tilde{f}_{0}$ is

$\left\langle\tilde{f}_{0}^{2}\right\rangle=\sigma^{2} \sum_{j=1}^{m} \frac{1}{j^{2}}=\frac{0.01^{2}}{m} \sum_{j=1}^{m} \frac{1}{j^{2}}$.

As a consequence, the average error on the estimation of $\tilde{f}_{0}$ is $\delta \phi_{\text {iso }} \simeq 0.005$. In the same spirit the errors on the density isocontours $\delta \rho_{\text {iso }}$ (Eq. (D.2)) are

$\delta \rho_{\text {iso }}^{2}=\frac{0.01^{2}}{m} \sum_{j=1}^{\tilde{m}}\left(j^{2}+1\right)^{2}$.

In practice the expansion in the above formula is conducted to order $\tilde{m}$, with $1<\tilde{m}<m$ in order to limit the amplitude of the 


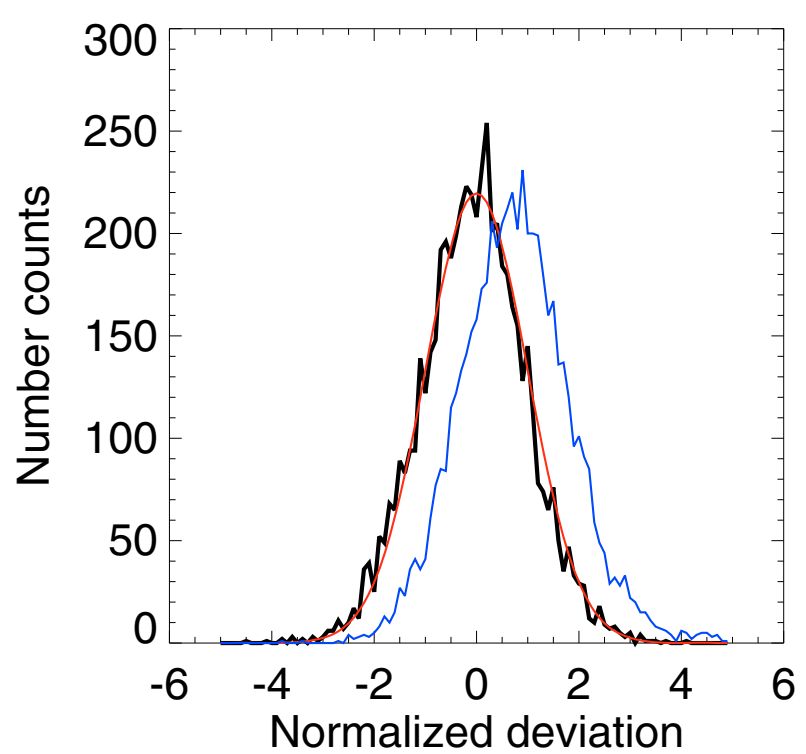

Fig. 8. Histogram of the difference image between the model and data. The black curve is the histogram of the pixels in the difference image. For each pixel the difference has been weighted by the local Poisson noise expectation. The red curve is the theoretical Gaussian expectation for the normalized residuals. The closeness between the black and red curves indicates that the result of the fit is very close to optimal. The blue curve is the histogram of normalized residuals of the data without subtracting the model. This curve is plotted here for reference; it illustrates the effect of subtracting the model from the data.

noise. Some numerical experiments shows that for $\tilde{m}>4$ there is no significant changes to the iso-contour shape, while on the other hand the noise grows quickly with $\tilde{m}$. Thus taking $\tilde{m}=4$, the resulting error is $\delta \rho_{\text {iso }}=0.077$.

\section{Prediction of image color}

Figure 9 indicates that the last image (lower right position in Fig. 1) does not have the typical "hat-shaped" color diagram observed for the 3 other images. But this feature is actually a prediction of the perturbative model. The model predicts that the first half of the last image comes from the same source area as the other images. The other half of the last image corresponds to an area of the source that has no counterpart in the large arcs, and only a tiny counterpart in the lower left image. This situation is due to the crossing of the caustic line by a faint part of the source which is visible in Fig. 9 in the lower left of source-caustic diagram. The part of the source situated inside the caustics corresponds to the first half of the last image, while the other half of this image corresponds to the faint part of the source outside the caustics. As a consequence, the first half of the last image should have the same color diagram as the other images, and this is exactly what is observed (see Fig. 9). The remaining part should have the same color as the faint parts of the source, and this is also what Fig. 9 illustrates. The color variations in the source are entirely related to the different colors of the bright and faint parts as illustrated in Fig. 9. The accurate prediction of the observed image colors is a confirmation of the model.

\section{Shape of the dark component}

The shape of the potential iso-contour is given by Eq. (C.1). However, in practice, only $\tilde{f}_{0}$ is known, and Eq. (7) shows that the first order coefficients of $\tilde{f_{0}}$ depend also on the impact parameters. But these first order terms are related to the centering of the potential, not to its shape, for instance the ellipticity is related to order 2 Fourier terms. It is however possible to obtain some partial information about the centering of the potential by using Eq. (B.3) the first order moments within the unit circle do not depend on the impact parameters (they cancel out in the calculations). The numerical value of the inner moments is quite small, of the order of $0.1 R_{\mathrm{C}}$. The outer moments have to be of the same order, and thus the centering terms are not large. Note that equations have been normalized by the factor $\kappa_{2}$ which depends on specific properties of the background. In practice $\kappa_{2}$ acts as an unknown scaling factor on $\tilde{f}_{0}$ and thus on the potential iso-contours. However, an important point is that this scaling degeneracy does not affect the shape of the potential, and for realistic halos $\kappa_{2}$ is close to unity (Peirani et al. 2008). The potential iso-contours $\mathrm{d} r=-\tilde{f}_{0}$ are presented in Fig. 10.

\subsection{Mass to light relation}

The un-perturbed potential iso-contour is a circle with radius $r=1$ when a local perturbation is introduced, some distortions to the circular iso-contours will be visible in the neighborhood of the perturbator. As a consequence, provided that the potential is generated by the visible matter, the distortions should correlate with the position of the galaxies. Since the center of the potential is unknown, the center of the un-perturbed unit circle is also unknown, and will have to be adjusted. The adjustment of this local circle is performed in the neighborhood of points on the potential iso-contour. The angular range of the circle adjustment is $\frac{\pi}{2}$. The residual of this local unit circle adjustment is presented in Fig. 10. The local deviations from circularity are not consistent with the positions of the galaxies. The opposite is visible; the maximum deviations from circularity are observed in the lower part of the figure where no nearby galaxies are present. The average deviation from circularity of the potential in this area is $\simeq 0.05 R_{\mathrm{C}}$. According to the mean error on the potential estimated in Sect. 4.6.3, this is a $10 \sigma$ deviation. This result demonstrates the need for a dark component that does not follow light. This is an interesting point for theories that try to avoid dark matter by modifying gravity. A natural solution is to consider that the dark component was formed by merging of cold dark matter halos.

\subsection{Inner and outer contribution to the potential}

Note also that Eq. (B.3) relates the Fourier coefficients of the fields $\frac{\tilde{f}_{0}}{\mathrm{~d} \theta}$ and $\tilde{f}_{1}$ to the inner $\left(a_{n}, b_{n}\right)$ and outer terms $\left(c_{n}, d_{n}\right)$ of the multipole expansion. As a consequence, the expansion of $\tilde{f}_{0}$ can be separated into inner contributions (which is equivalent to taking $c_{n}=d_{n}=0$ in the original expansion), and outer terms $\left(a_{n}=b_{n}=0\right)$. A numerical estimation shows that about $75 \%$ of the total power spectrum of the field $\tilde{f}_{0}$ comes from inner terms, which demonstrates clearly that the observed effects are dominated by the inner contributions. Since the deviation from circularity of the potential in areas with no light are much above the noise $(10 \sigma)$, the result that mass does not follow cannot be affected by the $25 \%$ contribution of the outer terms.

\subsection{Density}

To estimate the density of the lens it is necessary to make some assumptions. Making a specific assumption about the background allows us to make an estimation of $\kappa_{2}$ and also break the 

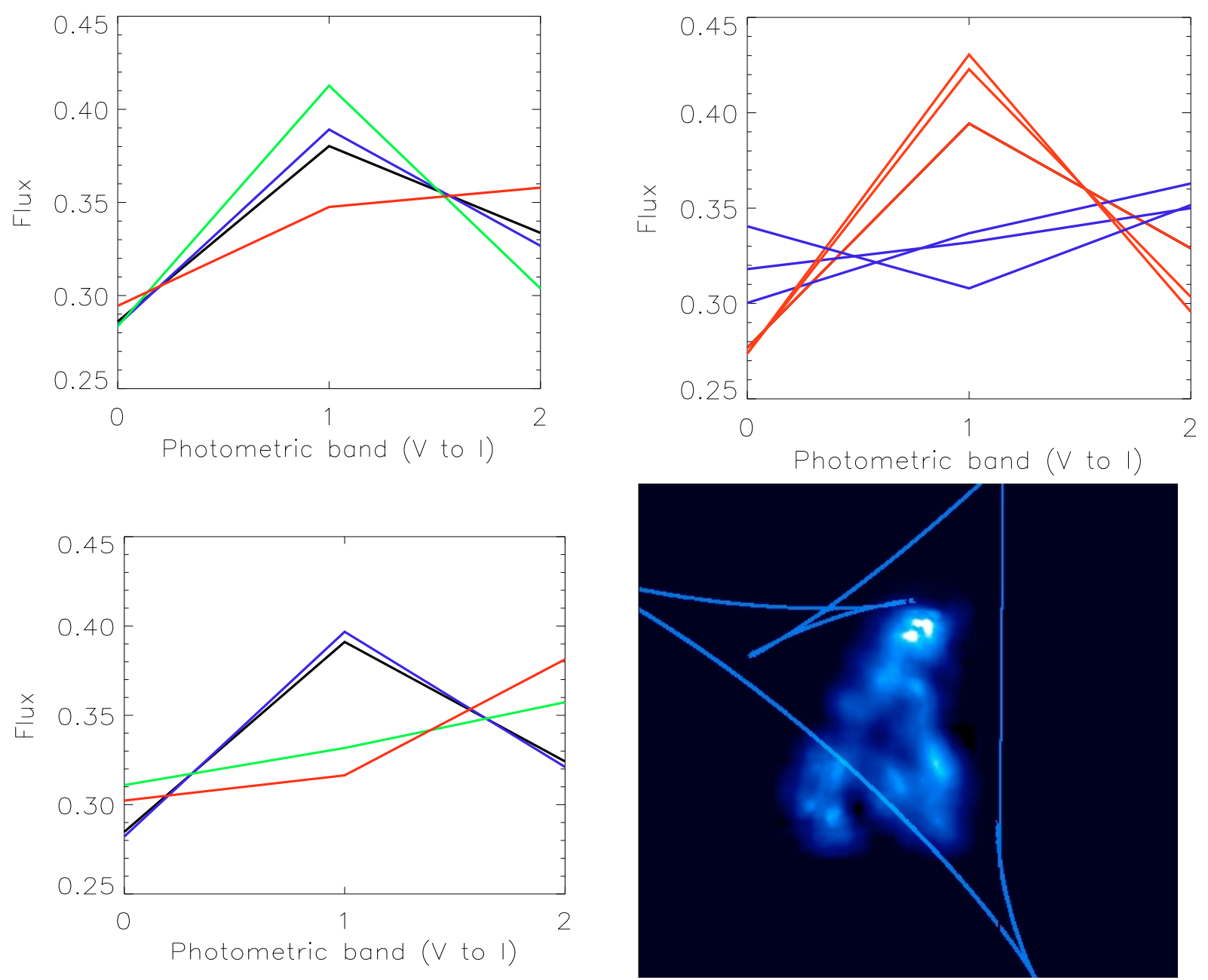

Fig. 9. The 3 color diagrams obtained by taking the sequence of raw flux in the 3 HST bands, as well as the source-caustic system (lower right). The upper left presents the color digram of the last image (red), and of the 3 other images. The upper right presents the color diagram for the bright parts of the first 3 images (red curves), while the blue curves represent the faint parts of the source. The lower left plot, compares the color diagram of the first half of the last image (black curve), to the mean diagram of the 3 other images (blue curve), and also compares the color diagram of the second half of the last image (green curve), to the mean color diagram of faint source parts in the 3 other images (red curve). The last figure presents the source and the system of caustics in the source plane.

mass-sheet degeneracy. For nearly isothermal lens distributions, the density iso-contours are given by Eq. (D.2). Note that in this equation the terms corresponding to the impact parameters cancel out

$\mathrm{d} r_{i}=\frac{\mathrm{d}^{2} f_{0}}{\mathrm{~d}^{2} \theta}+f_{1}=\frac{\mathrm{d}^{2} \tilde{f}_{0}}{\mathrm{~d}^{2} \theta}+\tilde{f_{1}}$.

To optimize the signal to noise ratio, the iso-contours presented in Fig. 10 are reconstructed by using an order 4 Fourier expansion (see Sect. 4.6.3 for details). Note that a method to reconstruct the iso-contours of a nearly isothermal lens was also studied by Evans \& Witt (2003). However, their description of the potential is less general since it contains only one angular functional, it is suitable only for potentials with constant isophotes.

\section{Discussion}

This paper describes the structure of the dark matter envelope of a small group of galaxies. It is shown that the distribution of the dark mass does not follow light (Fig. 10). This is direct evidence for an independent dark component at the scale of galaxies. Such new results about a system with a mass much lower than a typical cluster (for instance the Bullet Cluster) are essential. First because there has always been a problem with missing baryonic mass in clusters (for instance $\mathrm{X}$ ray gas); second because structures like the Bullet Cluster contains many galaxies, and their distances are not known with good enough accuracy to avoid some degeneracy (Clowe et al. 2006). The galaxies in this small group have very much the same colors, and as a consequence are probably very nearly at the same distance. Interpretating these results is not ambiguous and we are probably observing a merging event between two cold dark matter halos. Since the dark matter halos are much more extended than the bright cores of the galaxies, the interaction between the dark halos is already quite strong, while at the same time the stellar distribution is much less affected. However, by looking carefully at the outer part of the luminous halo's of the galaxies, some indications of interaction are visible. In particular, the lack of asymmetry in the outer contours of the light distribution indicates that the small galaxy at the center of the group has probably been stripped of its outer 


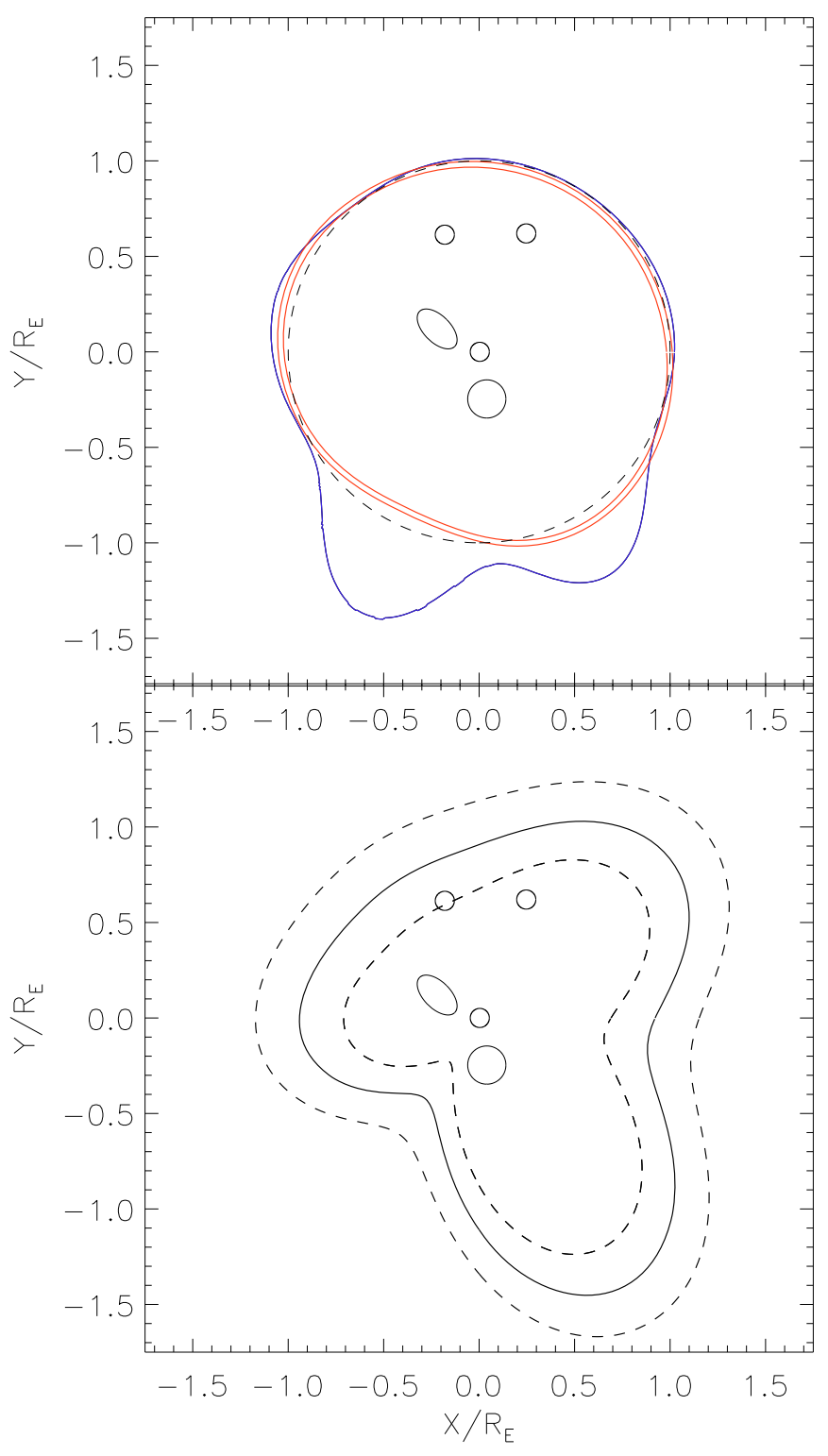

Fig. 10. The upper plot presents the potential iso-contours, the lower plot presents the density iso-contours. In the upper plot, the red curves represent the two $3 \sigma$ contours of the potential iso-contours. The dashed curve is the unit circle. The blue curve represents the local deviation from circularity of the potential. The distance between the blue curve and the unit circle is proportional to the deviation from circularity. The lower plot presents the two $3 \sigma$ iso-contours of the density. In each plot the small elliptical contours represent the galaxies in the lens. The small black contours represent the galaxies.

parts. This issue will be analyzed in more detail in a forthcoming paper. The analysis of this particular lens makes it clear that the combination of the perturbative method and good HST data open interesting new possibilities. Using this approach it should be possible to probe the structure of the dark component at the mass scale of galaxies for a large number of systems. Statistical results about the geometry and structure of the dark halos should be derived, and will probably offer the possibility to evaluate the amount of substructure in the halos (Alard 2008). It is clear also that the mass-luminosity relation could be analyzed for a number of other systems, and that the discovery of systems similar to this one would constrain even more a modification of gravity.
Acknowledgements. This work is based on HST data, credited to STScI and prepared for NASA under Contract NAS 5-26555. I would like to thank François Sevre, Leslie Sage, J. P. Beaulieu, S. Mao, R. Blandford, and P. Alard for helping with this paper, and the referee for helping to improve the presentation of this paper.

\section{Appendix A: Physical meaning of the perturbative approximation}

For circular sources, Eq. (8) shows that $\tilde{f}_{1}$ is the mean radial position of the image. The width of the image is $\sqrt{R_{0}^{2}-\left(\frac{\mathrm{d} \tilde{f}_{0}}{\mathrm{~d} \theta}\right)^{2}}$. The condition for image formation is $\left|\frac{\mathrm{d} \tilde{f}_{0}}{\mathrm{~d} \theta}\right|<R_{0}$, thus images are formed near the minimas of $\left|\frac{\mathrm{d} \tilde{f}_{0}}{\mathrm{~d} \theta}\right|$. The minimas of $\left|\frac{\mathrm{d} \tilde{f}_{0}}{\mathrm{~d} \theta}\right|$ are of two different types: linear behavior of $\frac{\mathrm{d} \tilde{f}_{0}}{\mathrm{~d} \theta}$ near the minima (small images), or a higher order polynomial behavior: caustics. To illustrate these properties, let's take the simple case of an elliptical potential, with ellipticity parameter $\eta$ :

$\phi=F\left(\sqrt{(1-\eta) x^{2}+(1+\eta) y^{2}}\right)$.

Let's now develop this potential to first order in $\eta$ :

$\phi \simeq F(r)-\frac{\eta}{2} F^{\prime}(r) r \cos 2 \theta$.

As a consequence, $\phi_{0}(r)=F(r), \psi(r, \theta)=\frac{\eta}{2} F^{\prime}(r) r \cos 2 \theta$ (see Eq. (4)) and since the background potential must be critical at $r=1, F^{\prime}(1)=1$. Using Eqs. (5) and (7) the perturbative fields reads:

$$
\left\{\begin{array}{c}
\tilde{f}_{1}=-\frac{\eta}{2}\left(1+F^{\prime \prime}(1)\right) \cos 2 \theta+x_{0} \cos \theta+y_{0} \sin \theta \\
\frac{\mathrm{d} \tilde{f}_{0}}{\mathrm{~d} \theta}=\eta \sin 2 \theta-x_{0} \sin \theta+y_{0} \cos \theta
\end{array}\right.
$$

Placing the source on the $X$-axis $\left(y_{0}=0\right)$, at an impact parameter $x_{0}=2 \eta$ Eq. (A.2) one obtains at $\theta=0: \frac{\mathrm{d}^{n} f_{0}}{\mathrm{~d} \theta^{n}}=0, n=1 . .3$, which corresponds to a broad minima of $\left|\frac{\mathrm{d} \tilde{f}_{0}}{\mathrm{~d} \theta}\right|$ near $\theta=0$ (see Fig. A.1). This minimum corresponds to the formation of an arc. This is a case of a cusp caustic, and $\frac{\mathrm{d} \tilde{f}_{0}}{\mathrm{~d} \theta}$ behaves like an order 3 polynomial near the singularity. The counter-image in the opposite direction is much smaller and corresponds to a narrow minimum of $\left|\frac{\mathrm{d} \tilde{f}_{0}}{\mathrm{~d} \theta}\right|$. For this small image $\frac{\mathrm{d}^{n} f_{0}}{\mathrm{~d} \theta^{n}}=0$ for $n=1$ only, and the behavior of this field is linear near the image.

\section{Appendix B: Relation to multipole expansion}

The expansion of the perturbative potential at a given radial position $r \psi$ reads (Kochanek 1991):

$$
\begin{aligned}
\psi= & -\sum_{n} \frac{a_{n}(r)}{r^{n}} \cos n \theta+\frac{b_{n}(r)}{r^{n}} \sin n \theta \\
& +c_{n}(r) r^{n} \cos n \theta+\mathrm{d}_{n}(r) r^{n} \sin n \theta .
\end{aligned}
$$

The perturbative theory requires the evaluation of the potential gradient on the critical circle. Since the coordinate system has been re-normalized so that the critical radius is situated at $r=1$, all the evaluation of quantities related to the potential will be 


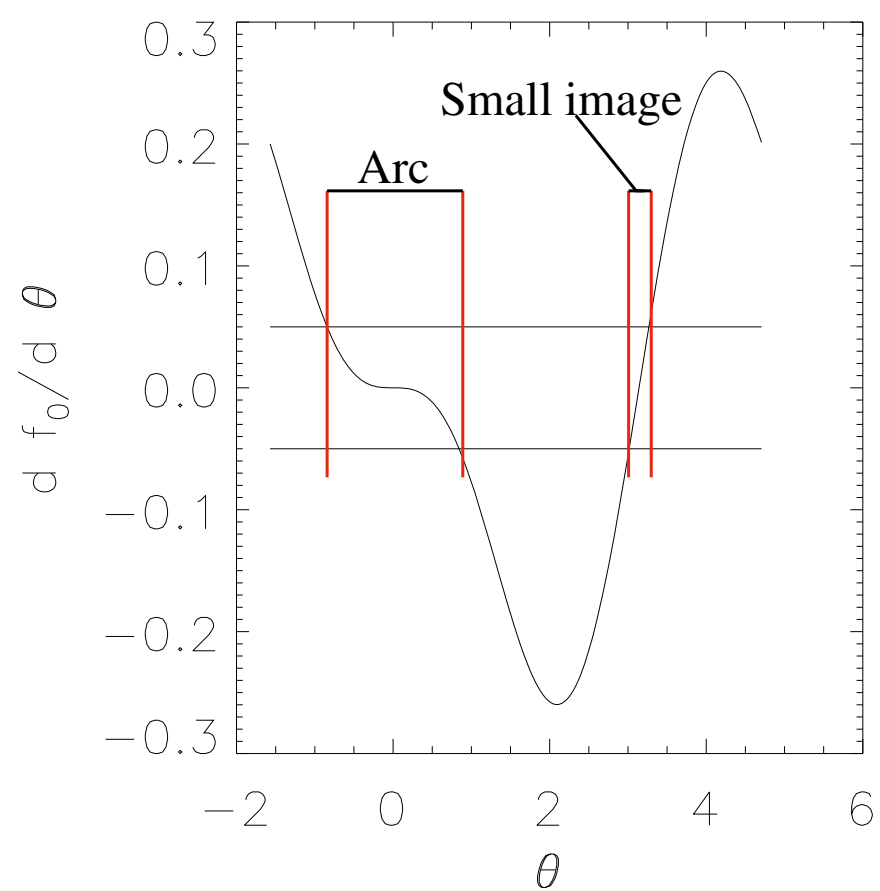

Fig. A.1. Plot of the function $\frac{\mathrm{d} \tilde{f}_{0}}{\mathrm{~d} \theta}\left(x_{0}=2 \eta\right.$ and $y_{0}=0$ in Eq. (A.2)). Images are formed when $\frac{\mathrm{d} \tilde{f}_{0}}{\mathrm{~d} \theta}<R_{0}$. The nature of the image is related to the behavior of $\left|\frac{\mathrm{d} \tilde{f}_{0}}{\mathrm{~d} \theta}\right|$ near its minima, the arc corresponds to a large minima, with cancellation of the first and second order derivatives of $\frac{\mathrm{d} \tilde{f}_{0}}{\mathrm{~d} \theta}$. For the small image, the behavior of $\frac{\mathrm{d} \tilde{f}_{0}}{\mathrm{~d} \theta}$ near zero is linear, and corresponds to a much smaller minima of $\left|\frac{\mathrm{d} \tilde{f}_{0}}{\mathrm{~d} \theta}\right|$.

performed at $r=1$. The coefficients $\left(a_{n}, b_{n}, c_{n}, d_{n}\right)$ are related to the projected density of the lens $\rho$ by the following formula:

$\left\{\begin{array}{l}a_{n}=\frac{1}{2 \pi n} \int_{0}^{2 \pi} \int_{0}^{r=1} \rho(u, v) \cos n v u^{n+1} \mathrm{~d} u \mathrm{~d} v \\ b_{n}=\frac{1}{2 \pi n} \int_{0}^{2 \pi} \int_{0}^{r=1} \rho(u, v) \sin n v u^{n+1} \mathrm{~d} u \mathrm{~d} v \\ c_{n}=\frac{1}{2 \pi n} \int_{0}^{2 \pi} \int_{r=1}^{\infty} \rho(u, v) \cos n v u^{1-n} \mathrm{~d} u \mathrm{~d} v \\ d_{n}=\frac{1}{2 \pi n} \int_{0}^{2 \pi} \int_{r=1}^{\infty} \rho(u, v) \sin n v u^{1-n} \mathrm{~d} u \mathrm{~d} v .\end{array}\right.$

Using Eqs. (B.1) and (B.2), and noting that: $\left(\frac{\mathrm{d}\left(a_{n}+c_{n}\right)}{\mathrm{d} r}\right)_{[r=1]}=$ $\left(\frac{\mathrm{d}\left(b_{n}+d_{n}\right)}{\mathrm{d} r}\right)_{[r=1]}=0$ the fields $\frac{\mathrm{d} f_{0}}{\mathrm{~d} \theta}=\left(\frac{\partial \psi}{\partial \theta}\right)_{[r=1]}$ and $f_{1}=\left(\frac{\partial \psi}{\partial r}\right)_{[r=1]}$ :

$\left\{\begin{array}{c}f_{1}=\sum_{n} n\left(a_{n}-c_{n}\right) \cos n \theta+n\left(b_{n}-d_{n}\right) \sin n \theta \\ \frac{\mathrm{d} f_{0}}{\mathrm{~d} \theta}=\sum_{n}-n\left(b_{n}+d_{n}\right) \cos n \theta+n\left(a_{n}+c_{n}\right) \sin n \theta .\end{array}\right.$

As a consequence, there is a direct relation between the Fourier expansion of the perturbative fields and the multipole expansion of the potential at the critical circle. This relation also demonstrates that the Fourier expansion of the perturbative fields is not equivalent to the Fourier expansion of the potential by Trotter et al. (2000). The expansion proposed by Trotter requires a number of additional coefficients with respect to the multipole expansion. On the other hand, Eqs. (B.1)-(B.3) indicate that the perturbative expansion is directly related to the multipole expansion without needing any additional coefficients. It is also clear from Eqs. (B.1) and (B.3) that since the Fourier expansion of elliptical potentials is approximately of order 2 (see Eq. (A.1)), the corresponding Fourier expansion will also be of order 2. Higher order terms in the Fourier expansion of the perturbative fields will thus be related to the skewness, boxyness, etc., of the potential. The first order terms are related to the centering of the potential.

\section{Appendix C: Geometrical interpretation}

The perturbative fields are directly related to the geometrical structure of the potential. The potential iso-contour near the critical circle is easy to infer from Eq. (D.1). The iso-contour equation reads:

$\phi_{0}(r)+\epsilon f_{0}(\theta)+\epsilon f_{1}(\theta)(r-1)=C$.

Searching for the iso-contour near the critical circle $r_{i}=1+\epsilon \mathrm{d} r_{i}$ and developing the former equation to first order in $\epsilon$ :

$\epsilon\left(\mathrm{d} r_{i}+f_{0}\right)=C-\phi_{0}(1)$.

When no perturbation is introduced $\left(f_{0}=0\right)$, the iso-contour must be reduced to the critical circle $(\mathrm{d} r=0)$, as a consequence the right side of the equation must be zero too, and the former iso-contour equation reduces to:

$\mathrm{d} r_{i}=-f_{0}$.

The potential iso-contour depend only on $f_{0}$, while the isocontour variation depends also on $f_{1}$. Considering a contour with radial position $r_{i}$, defined by $\phi\left(r_{i}, \theta\right)=C$, the equation of a nearby contour is $\phi\left(r_{i}+\delta r_{i}, \theta\right)=C+\mathrm{d} C$, or equivalently $\frac{\mathrm{d} \phi}{\mathrm{d} r}\left(r_{i}, \theta\right) \delta r_{i}=d C$. There is no iso-contour variation provided that $\delta r_{i}$ is constant, which is equivalent to $\frac{\mathrm{d} \phi}{\mathrm{d} r}\left(r_{i}, \theta\right)$ being constant. To first order in $\epsilon$ :

$\frac{\mathrm{d} \phi}{\mathrm{d} r}\left(r_{i}, \theta\right) \simeq 1+f_{1}-\frac{\mathrm{d}^{2} \phi_{0}}{\mathrm{~d}^{2} r}(1) f_{0}$.

Considering that Eq. (C.2) depends only on $f_{1}$ for purely isothermal backgrounds, and that CDM halos are quite close to isothermal in general, the iso-contour variation is dominated by $f_{1}$.

\section{Appendix D: Relation to the geometry of the density}

The two perturbative fields are related to the potential by the following expansion (Eq. (4)):

$\phi=\phi_{0}(r)+\epsilon f_{0}(\theta)+\epsilon f_{1}(\theta)(r-1)$.

Note that since $r=1+\epsilon \mathrm{d} r$ the expansion presented in Eq. (D.1) is of order 2 in $\epsilon$. This expansion reduces to order 1 when introduced in the lens equation. By inserting Eq. (D.1) in the Poisson equation, taking $r=1+\epsilon \mathrm{d} r$, and developing to first order in $\epsilon$, the density reads

$\rho \simeq\left(1+2 C_{2}\right)+\left(\left(2 C_{2}+6 C_{3}-1\right) \mathrm{d} r+\frac{\mathrm{d}^{2} f_{0}}{\mathrm{~d}^{2} \theta}+f_{1}+2 f_{2}\right) \epsilon$.

The iso-contour near the critical circle is defined by the equation $\rho=C$. In the absence of a perturbation $\left(f_{i}=0\right)$ the iso-contour must reduce to the critical circle $(\mathrm{d} r=0)$. As a consequence the equation of the iso-contour is:

$\left(1-2 C_{2}-6 C_{3}\right) \mathrm{d} r_{i}=\frac{\mathrm{d}^{2} f_{0}}{\mathrm{~d}^{2} \theta}+f_{1}+2 f_{2}$.

For a nearly isothermal distribution, the higher order derivatives $(n>1)$ are close to zero. Assuming that these higher order derivatives are of order $\epsilon\left(C_{2} \simeq C_{3} \simeq \epsilon\right.$ and $\left.f_{2} \simeq \epsilon F(\theta)\right)$, the former equation reduce to:

$\mathrm{d} r_{i}=\frac{\mathrm{d}^{2} f_{0}}{\mathrm{~d}^{2} \theta}+f_{1}$ 


\section{References}

Alard, C. 2000, A\&AS, 144, 363

Alard, C. 2007, MNRAS, 382, L58

Alard, C. 2008, MNRAS, 388, 375

Blandford, R. D., \& Kovner, I. 1988, Phys. Rev. A 38, 4028

Brewer, B., \& Lewis, G. 2006, ApJ, 637, 608

Cabanac, R. A., Alard, C., Dantel-Fort, M., et al. 2007, A\&A, 461, 813

Clowe, D., Bradač, M., Gonzalez, A. H., et al. 2006, ApJ, 648, L109

Diego, J. M., Protopapas, P., Sandvik, H. B., et al. 2005, MNRAS, 360, 477

Evans, N., \& Witt, H. 2003, MNRAS, 345, 1351

Grossman, S., \& Narayan, R. 1988, ApJ, 324L, 37

Kochanek, C., Blandford, R., Lawrence, C., et al. 1989, MNRAS, 238, 43

Krist, J. 1995, ASPC, 77, 349

Limousin, M., \& the SL2S collaboration 2008 [arXiv:0812 . 1033]

Lynds, C., \& Petrosian, V. 1986, BA\&AS, 18, 1014
Nelder, J., \& Mead, R. 1965, Comp. J., 7, 308

Paczyński, B. 1987, Nature, 325, 572

Peirani, S., Alard, C., Pichon, C., Gavazzi, R., \& Aubert, D. 2008, MNRAS, 390, 945

Press, W., Teukolsky, S., Vetterling, W., et al. 2007, Numerical Recipes (Cambridge University Press)

Saha, P., \& Read, J. 2009, ApJ, 690, 154

Suyu, S., Marshall, P., Hobson, M., et al. 2006, MNRAS, 371, 983

Suyu, S., Marshall, P., Blandford, R., et al. 2009, ApJ, 691, 277

Trotter, C., Winn, J., \& Hewitt, J. 2000, ApJ, 535, 671

Van Cittert, P. H., \& Physik, Z. 1931, 69, 298

Vegetti, S., \& Koopmans, L. 2009, MNRAS, 392, 945

Wallington, S., Kochanek, C., \& Narayan, R. 1996, ApJ, 465, 64

Warren, S., \& Dye, S. 2003, ApJ, 590, 673

Wayth, R., Warren, S., Lewis, G., et al. 2005, MNRAS, 360, 1333

Wucknitz, O., Biggs, A., \& Browne, I. 2004, MNRAS, 349, 14 